Aus dem pathologisch-anatomischen Institute und der dermat. Klinik in Wien.

\title{
Ein weiterer Beitrag zur Aetiologie des Ecthyma gangraenosum.
}

Von

\section{Dr. Fritz Hitschmann und Dr. Karl Kreibich.}

(Hierzu Taf. VII u. VIII.)

In Nr. 50 "Wiener klinische Wochenschrift" 1897 beschrieben wir zwei Fälle von Ecthyma gangraenosum und hielten uns auf Grund unserer bakteriologischen und histologischen Untersuchungen zu dem Schlusse berechtigt: Der Bacillus pyocyaneus ist in unseren beiden Fällen der Erreger des Ecthyma gangraenosum, besitzt somit die Eigenschaften eines für den Menschen pathogenen Bakteriums. In ihren klinischen Erscheinungen wichen damals beide Fälle von einander ab. Während im ersten Falle die Hautaffection aus linsengrossen, runden, schmutzig röthlichen, derben Papeln mit lebhaftem rothen Halo bestand, stellten im zweiten Falle die Efflorescenzen haselnussgrosse, blaurothe, scheinbar furunkelartige Infiltrate mit centralem haemorrhagisch nekrotischen Zerfalle dar. Daneben fanden sich in diesem Falle auch zwei kreuzergrosse, leicht unter das Niveau eingesunkene Stellen nit dunkelbraunrother haemorrhagisch nekrotischer Oberfläche ohne tieferes Infiltrat.

Aus den Beschreibungen Hallopeau's entnahmen wir dann, dass nach Einreissen der Epidermis aus den papulösen Efflorescenzen, napfförmige mit Detritus belegte Geschwüre mit steilen Rändern und entzündlichem Halo sich bilden, die nach Vidal zu grösseren Herden confluiren können. 
Auch H. Neumann, Ehlers, Oettinger, Williams und Karlinski erblickten das Wesentliche des Processes in einem umschriebenen Entzündungsøorgang ${ }^{1}$ ) der Haut, mit frühzeitiger centraler haemorrhagischer Nekrose und consecutiver Geschwürsbildung.

Die Affection findet sich rorwiegend bei Kindern, aber auch hier erst dann, wenn durch vorausgegangene Erkrankungen wie Enteritis, Tuberculose etc. der Organismus geschwächt wurde. In gleicher Weise fand man die Erkrankung nach schweren fieberhaften Erkrankungen (Typhus, Phlegmone) vereinzelt auch bei Erwachsenen.

Wir waren damals der Ansicht, dass eine idiop ath is che und symptomatische Form des Ecthyma gangraenosum zu unterscbeiden sei und dass letztere auftrete im Verlaufe der durch den Bacillus pyocyaneus bewirkten Allgemeininfecti io n, einer schweren, fieberhaften, gewöhnlich zum Exitus letalis führenden Allgemeinerkrankung.

Auch wir fanden in unserem zweiten Falle ausser den Erscheinungen anf der Haut, ausgedehnte Blutungen in der Zunge, im Larynx und in der Lunge, hielten dieselben aber nicht für Zeichen einer Allgemeinerkrankung, sondern für eine fortgeleitete Infection von der erkrankten Mundhöhle aus.

In diesen Blutungen nun, sowie in den Efflorescenzen der Haut fand sich in reicher Menge ein Stäbchen, das culturell sehr leicht als Bacillus pyocyaneus zu erkennen war. Schon Ausstrichpräparate aus den Hautefflorescenzen ergaben in grosser Anzahl den dünnen Bacillus, der sich dann in den Schnitten der erkrankten Haut, dieselbe dicht durchsetzend, die Gefässe umlagernd wieder fand. Die Reincultur des Bakteriums auf Thiere übertragen, bewirkte Blutungen in den Lungen und serösen Häuten; an der Inoculationsstelle, Haemorrhagien, Nekrose und geringe Entzündungserscheinungen, kurz jenen Process, der die Hautefflorescenzen charakterisirte. Die nekrotischen Erscheinungen im Ecthyma gangr. scheinen gebunden an die Menge der Bakterien, so dass die Vermuthung nahe lag, die Nekrose als eine dem Pyocyaneus specifische Wirkung

1) Thatsächlich ist es eine primäre Nekrose. 
zu betrachten. Auch die Haemorrhagien, die klinisch jene blaurothe oder braunrothe Beschaffenheit der Efflorescenzen bedingen, die fast übereinstimmend von allen Autoren beschrieben wird, erscheinen für die Einwirkung des B. pyocyaneus pathognomonisch. Bezüglich des Infectionsmodus hielten wir das Eindringen des Bakteriums durch die vorher durch Urin etc. macerirte Haut für wahrscheinlich; bezüglich der Infection der Lunge nahmen wir den bereits angedeuteten Modus an; für die Annahme einer Allgemeininfection fehlte uns der Nachweis des Bakteriums im Blate.

Seit dem Erscheinen unserer Untersuchungen wurden über den gleichen Gegenstand nur zwei Publicationen aus der Klinik Escherich veröffentlicht, die mangels literarischer Angaben auf einen Vergleich mit unseren Befunden nicht eingingen. Der erste von Blum als "Pyocyaneus-Septikaemie mit complicirender Pyocyaneus-Endocarditis im Kindesalter" beschriebene Fall ist kurz folgender: Bei einem $2{ }^{1}{ }_{2}$ Monate alten, hereditär luetischen Kinde treten unter hohem Fieber $(39 \cdot 4)$ prall gefüllte, blutige.Blasen an der ersten und zweiten Zehe des rechten Fusses auf, zu gleicher Zeit am Herzen ein lautes, rauhes systolisches Geräusch. Exitus letalis am zweiten Tage der fieberhaften Erkrankung. Die Section ergibt Pneumonia lobularis, Catarrhus intest. chron. und Hepatitis interstitialis luetica. Die Bicuspidalklappen an ihren Rändern verdickt und an ihren oberen Flächen miliengrosse Knötchen tragend. Aehnliche Knötchen in der vergrösserten braunrothen Leber. Das Herzblut, intra vitam und 1 $1 / 2$ Stunden post mortem untersucht, ergibt den B. pyocyaneus in Reincultur. Schnitte der Milz, Leber, Nieren, Lungen und Darm zeigen das Bakterium in wechselnder Menge. Jene milienartigen Knötchen an der oberen Fläche der Bicuspidalklappe bestehen aus Leukocyten und Bakterienhaufen.

Als Ausgangspunkt für die Septikaemie wird der Darm angenommen und als begüstigendes Moment der wahrscheinlich durch die hereditäre Lues bedingte schlechte Allgemeinzustand des Kindes betont.

Während nun Blum über die Untersuchung der beiden blutigen Blasen am rechten Fusse keine weiteren Angaben 
macbt, fand Escherich in seinem ersten Falle, einem cachektischen Kinde von 4 Wochen, am Oberschenkel einen grossen Abscess, dessen Spitze hämorrhagisch schwarz verfärbte Haut zeigt und dessen Eiter beim Verbandwechsel eine blaugrüne Verfärbung der Verbandstoffe aufwies. Der aus dem Abscesseiter reincultivirte B. pyocyaneus fand sich auch im Blut, Harn, Stuhl und Cerebrospinalflüssigkeit.

Unter den Erscheinungen einer Pneumonie geht das Kind zugrunde und die Section ergibt neben chronischem Darmkatarrh die bereits kliniscb diagnosticirte Pneumonie, in welcher sich culturell und im Schnitte der B. pyocyaneus nachweisen lässt. Während dieser Fall, trotz des Umstandes, dass die Anwesenheit des B. pyocyaneus erst beim Verbandwechsel constatirt wurde, als eine durch den genannten Bacillus bewirkte Allgemeininfection angesehen werden muss, hält Escherich dieselbe Erkrankung bei seinem zweiten Falle, einem abgemagerten Kinde von 2 Monaten, nur für wahrscheinlich. Mit Rücksicht darauf, dass der Nachweis des B. pyocyaneus im Blut, in der Milz und in den Nieren nicht gelang, hat dieser Fall für die Aetiologie des B. pyocyaneus nur insoferne eine Bedeutung, als er unter den äusseren Umständen einer Hausinfection entstand und ein Exanthem mit hämorrhagischen Charakter darbot. Vielleicht hätte gerade die Untersuchnng der Hautefflorescenzen den positiven Nachweis ergeben.

In den zwei letzten Fällen, zwei zum Exitus letalis führenden Brechdurchfällen, schliesst Es cherich selbst die pathogene Wirkung des im Darminhalte nachgewiesenen B. pyocyaneus aus.

Weitere Mittheilungen über Pyocyaneusinfection fanden wir in der Literatur nicht. Der Grund dafür liegt wohl einerseits in der grossen Selteuheit derselben, andererseits in dem Umstand, dass die Hautaffection in ihren klinischen Erscheinungen keineswegs schon vollkommen beschrieben ist.

Im Folgenden ein weiterer Beitrag zur Klinik und Anatomie der von uns als "Ecthyma gangraenosum" bezeichneten Hautaffection: Hochgradig abgemagertes Kind von 6 Wochen. Die fast rollständig fettarme Haut an einzelnen Stellen leicht. exfoliirt, im übrigen glänzend oder fein lamellig schuppend. 
In der Gegend um den After, an beiden Oberschenkeln (Beuge und Streckseite), in beiden Kniekehlen, ferner am Abdomen finden sich etwa 25-30 Efflorescenzen von gleichem Charakter, sich nur durch die verschiedene Grösse unterscheidend. Die Efflorescenzen stellen sich dar als dunkelbraune oder rothbraune, im Niveau der Haut gelegene, ziemlich scharf umschriebene Flecken von Linsen- bis Kreuzergrösse. Das Centrum der Flecken ist exfoliirt und mit einem grauen oder mehr gelblichgrauen, offenbar ron nekrotischem Gewebe herrührenden Detritus belegt. An einigen Effiorescenzen ist dieser centrale Antheil leicht eingesunken und die Umgrenzung dadurch scheinbar etwas elevirt.

Nach Entfernung dieses grauen, nekrotischen Detritus zeigt sich ein hämorrhagisch infiltrirtes, morsches Gewebe. An den kleineren, in ihrer Farbe etwas lebhafter rothen Efflorescenzen, ist der Beginn des centralen Zerfalles eben durch eine weisslich graue Verfärbung angedeutet. Der Mangel eines echten peripheren Infiltrates liess uns die noch in Betracht kommende Diagnose "Lues" leicht ausschliessen und die Diagnose „Ecthyma gangraenosum“ stellen.

Zur bakteriologischen Untersuchung wird eine Efflorescenz nach entsprechender Reinigung eingeschnitten; auf dem Durchschnitte ist trockenes und brüchiges, aber nirgends verflüssigtes Gewebe zu sehen. Durch Abstreifen mit dem Messer wird spärliches Material gewonnen, das der mikroskopischen und culturellen Untersuchung zugeführt wird.

Im mikroskopischen Präparate sieht man massenhaft dünne, mässig lange Stäbchen einer Art, die sich mit allen Anilinfarbstoffen gut tingiren und beim Verfahren nach Gram den Farbstoff wieder abgeben. Agarplatten mit demselben Materiale beschickt, zeigen nach 24stündiger Bebrütung Reinculturen desselben Stäbchens; wir bestimmten den Bacillus nach seinem Verhalten auf der Agarplatte, Gelatine, Fleischbrühe u. s. f. als Bacillus pyocyaneus.

Die Section des am nächsten Tage verstorbenen Kindes war aus rituellen Gründen nicht möglich, so dass dieser Fall für die Frage, ob wir es mit einer idiopathischen Form der 
Hautaffection oder einer Allgemeininfection mit consecutivem Exanthem zu thun hatten, keine Entscheidung bringt.

Die histologische Untersuchung mehrerer excidirter Efflorescenzen bestätigte vollständig unseren seiner Zeit gegebenen Befund. Es bandelt sich auch hier um ziemlich scharf umschriebene Herderkrankungen, die nach allen Richtungen ziemlich scharf absetzen. Es erstreckt sich die Veränderung in gleichem Masse auf Epidermis, Cutis und subcutanes Gewebe; die Zellen haben im Bereiche des Herdes mehr minder ihre Kernfärbbarkeit verloren, das Protoplasma färbt sich diffus mit dem Kernfarbstoffe. Daraus resultirt eine verwaschene, undeutliche Gewebsstructur. Inmitten des nekrotischen Gewebes sind einzelne Inseln mit wohlerhaltenen Zellen zu sehen; sie gehören Drüsen und Follikeln an. Die Gefässe sind hyperämisch; an zahlreichen Stellen kleinere und grössere Hämorrhagien. Die dem Herde zunächst gelegenen Papillen der Peripherie sind in ihrer ganzen Breite hämorrhagisch infarcirt, die weiter entfernten Papillen besitzen sehr stark hyperämische Gefässe. So entsteht der Halo.

Die Zellen der Epidermis sind, soweit die letztere über dem Herde erbalten ist, in den oberflächlichen Schichten von einem vollständigeren Kernverluste betroffen, als in den tieferen. Oder es fehlt die ganze Epidermis und mit ihr ein Theil des cutanen Gewebes, so dass das Cutisgewebe, oft aufgefasert frei liegt. Das Cutisgewebe zeigt ausserdem, dass der Process gegen die Tiefe zu an Intensität abnimmt, indem in den oberen Partien ein rollständiger Kernschwund besteht und das Gewebe jede Structur verloren und ein mehr minder homogenes Aussehen gewonnen hat. In den tieferen Partien sind die Kerne zum Theile erbalten, zum grösseren Theile schlecbt gefärbt oder fehlend und die Structur noch überall erkennbar.

Im Gewebe findet man hie und da zerstreut mässige Anhäufungen ron mono- und polynuclearen, oft im Zerfalle begriffener Leukocyten, die aber nirgends eine grössere Ausdehnung gewinnen. In der Cutis und im subcutanen Gewebe sind die Gefässe hyperämisch und das Gewebe ist von zahlreichen, zumeist kleineren Haemorrhagien durchsetzt. Um die Schweissdrüsen herum auch im Bereiche des Gesunden besteht ein mit Haemalaun sich lichtblau färbender Hof. 
Die Ausbreitung der Stäbchen ist in den mit polychromem Methylenblau gefärbten Schnitten fast dieselbe, wie in den früher beschriebenen Fällen; also auch hier liegt die Hauptmasse in der Epidermis, sie finden sich diffus zerstrent im ganzen übrigen erkrankten Gewebe, ohne aber jene Anhäufungen um die Gefässe herum zu bilden, wie wir sie seinerzeit abgebildet haben. Aber auch hier fehlt jegliches Vorkommen in den Gefässen. Nirgends waren andere Bakterien ausser diesen Stäbchen.

Vergleichen wir nun alle drei Fälle, so dürfen wir trotz einiger klinischer Verschiedenheiten das Ecthyma gangraenosum als eine wohl charakterisirte Erkrankung hinstellen.

Wir halten uns berechtigt, den eingangs citirten Schluss "der Bacillus pyocyaneus ist in unseren beiden Fällen der Erreger des Ecthyma gangraenosum" zu erweitern und zu behaupten: Bei hochgradig cachektischen Kindern findet sich in seltenen Fällen eine Erkrankung der Haut, die durch den Bacillus pyocyaneus erregt ist. Soweit die geringe Zahl von Beobachtungen eine Charakterisirung der Erkrankung zulässt, ist dieselbe gekennzeichnet durch eine meist scharf umschriebene, gewöhnlich von einem rothen Halo umgrenzte, rothbraune, oder mehr dunkelbraxune Verfürbung von Linsen- bis Kreuzergrösse. Das Centrum der Efflorescenzen zeigt frühzeitig hämorrhagisch nekrotischen Zerfall und die Sonde gelangt bei der Untersuchung in ein braunrothes, morsches, nicht blutendes Gewebe. Anatomisch entspricht diesen Efflorescenzen eine umschriebene Nekrose der Cutis mit reichlichen peripheren Hämorrhagien. Ausser Nekrose und Hämorrhagie finden sich noch die Zeichen einer geringgradigen Entzündung. Dort wo die Entzündungserscheinungen sehr geringgradig sind, liegt die Efflorescenz im Niveau der umgebenden Haut, bei etwas stärker ausgesprochener Entzündung treten die Efflorescenzen flach papelartig gegenüber der Umgebung hervor, wodurch die bereits erwähnte klinische Verschiedenheit erklärt wird. Aber auch bei diesen Efflorescenzen findet man die haemorrhagisch nekrotische Beschaffenheit des centralen Antheiles; letzteres Symptom zusammen mit demVorhandensein von reichlichen dünnen Stäbchen im Ausstrichpräparat dieses Antheiles halten wir für das wichtigste differential diagno- 
stische Moment, um diese Erkrankung, gegenüber klinisch ähnlichen, bei Kindern öfters zum centralen Zerfall der Efflorescenzen führenden Erkrankungen abzugrenzen. Die gewonnene Reincultur des B. pyocyaneus wird die klinische Diagnose „Ecthyma gangraenosum" vervollständigen.

In diesem Sinne wolle! wir noch ganz kurz über eine weitere Beobachtung berichten, die als Ecthyma zu spät erkannt. nur theilweise untersucht werden konnte. Es handelte sich um ein cachektisches, 3 (?) Monate altes Kind, das unter den Erscheinungen einer Pneumonie und eines chronischen Intestinalkatarrhes gestorben war. Bei der Obduction fand man auch einen chronischenDarmcatarrh und eine lobuläre Pneumonie. Ausser diesen Veränderungen fand man aber auch in der Haut zahlreiche hämorrhagisch nekrotische Efflorescenzen vor. Als nun aus den lobulär-pneumonischen Herden Pyocyaneusbacillen in Reinculturen aufgingen - mikroskopisch hatte man dünne, mässig lange Stäbchen gefunden - so erkannte man in jenen bämorrhagisch nekrotischen Efflorescenzen ein Ecthyma gangraenosum. Die Untersuchung der letzteren war aber leider nicht mehr möglich, da die Leiche mittlerweile beerdigt worden war.

Die histologische Untersuchung der Lunge ergibt eine typische lobuläre Pneumoniemit reichlichen Hämorrhagien. In denBakterien* schnitten sind diffus zerstreut mässig zahlreiche Stäbchen zu sehen.

Alle unsere Beobachtungen stammen aus der nied.-öst. Landesfindelanstalt; es ist natürlich den Anstaltsärzten schon lange das endemische Vorkommen des Pyocyaneus bekannt und blaugefärbte Verbände etwas alltägliches.

Wenn man aber früher mit dieser Blaufärbung des Verbandes die Rolle des Pyocyaneus als beendigt ansah und ihm nach dem damaligen Stande der Lehre nur eine saprophytische Existenz zuschrieb, so lehren unsere und Escherich's Beobachtungen, dass dem endemischen Vorkommen des Pyocyaneus eine pathologische Bedeutung zugesprochen werden muss, zumal es sich um Locale handelt, in denen sich kranke, fiir diese Infection prädisponirte Kinder aufhalten.

\section{Erklärung der Abbildungen auf Taf. VII u. VIII.}

Taf. VII. Abbildung eines Falls von Eethyma gangraenosum.

Taf. VIII. Fig. 1. Schnitt durch eine Ecthymaefflorescenz. Haem. Eosin. 1. Haemorrhagien. 2. Nekrose der Cutis. Fig. 2. 1. Bacillenanhäufung. 2. Nekrotische, fast kernlose Cutis. (Polychr. Methylenblau.) 


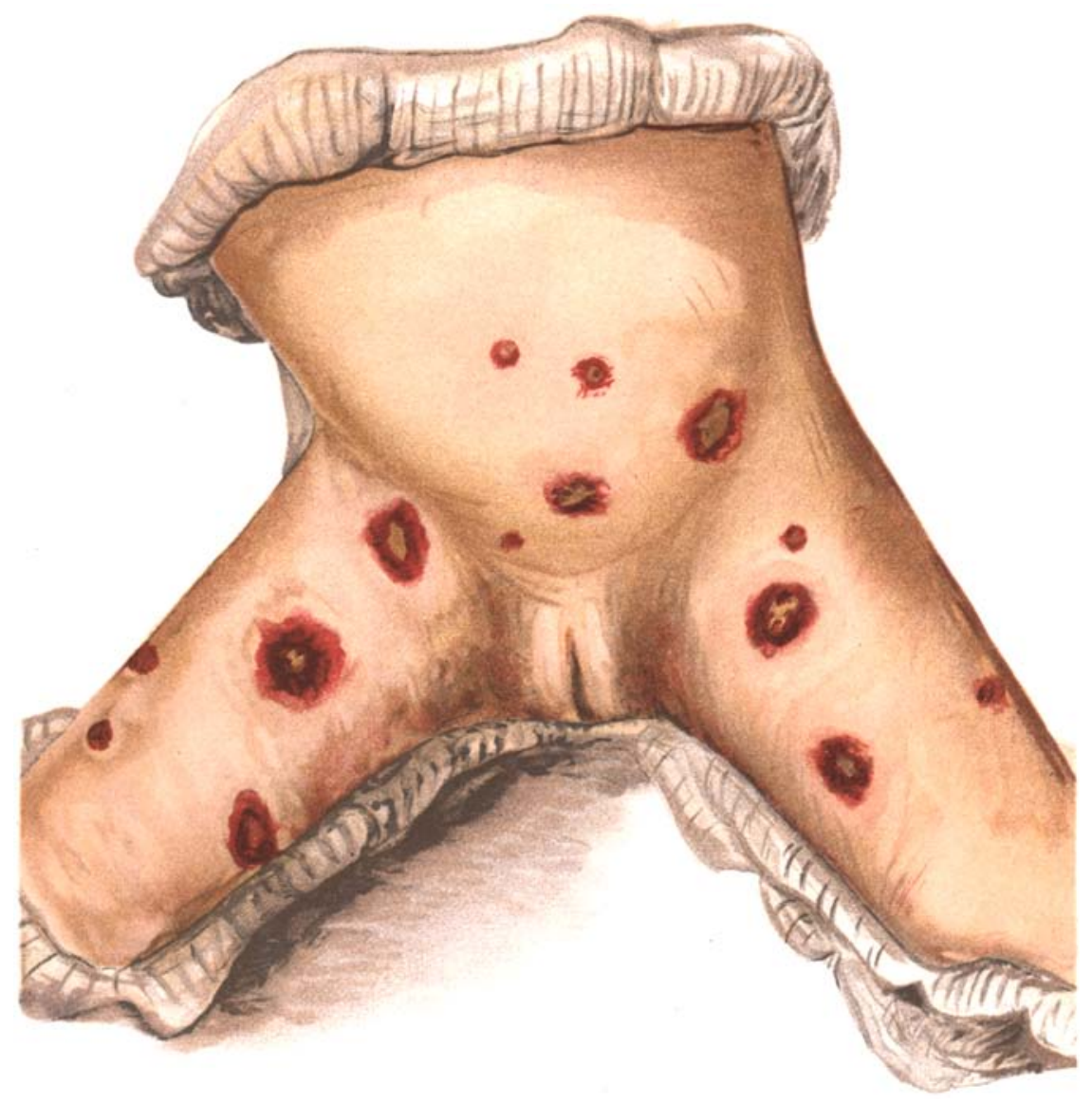

Hitschmann u. Keribich Ed fluma gangraenosum. 
Fig 1.

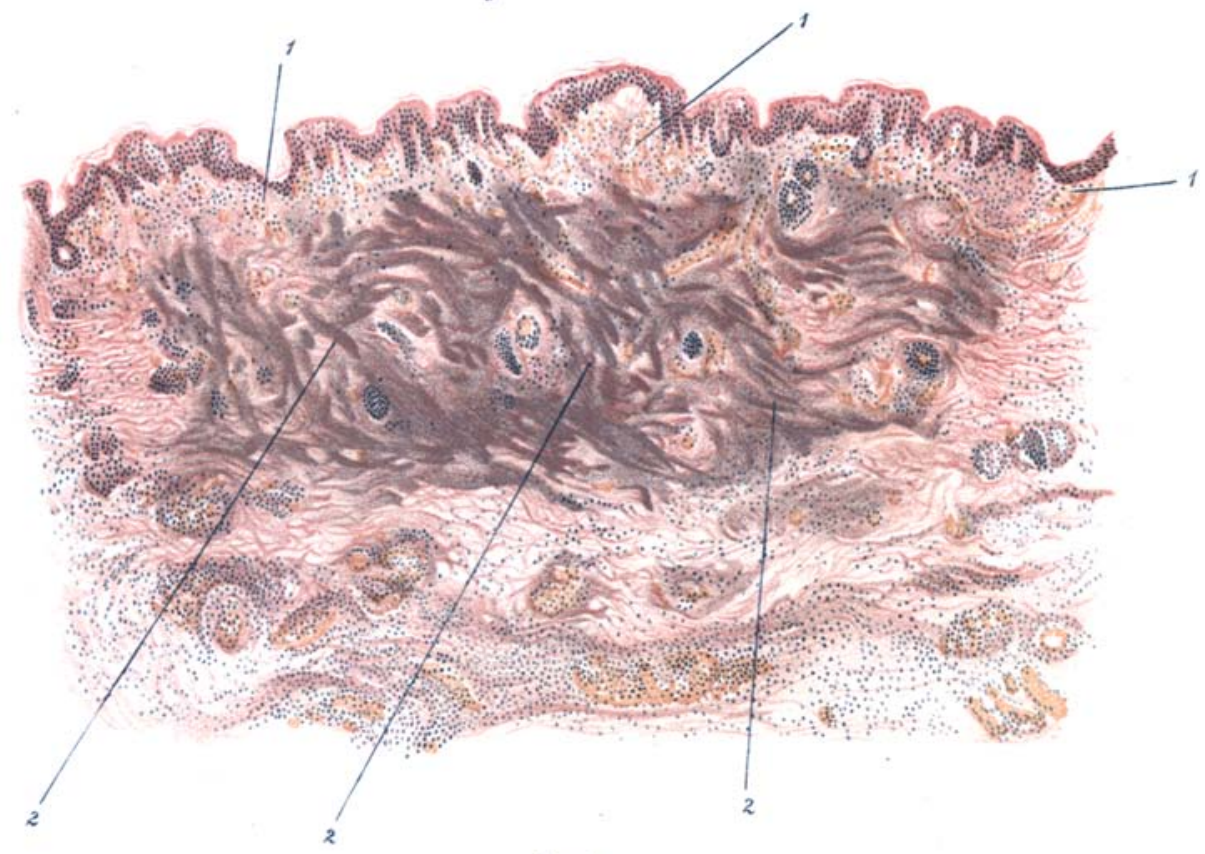

Fig. 2.

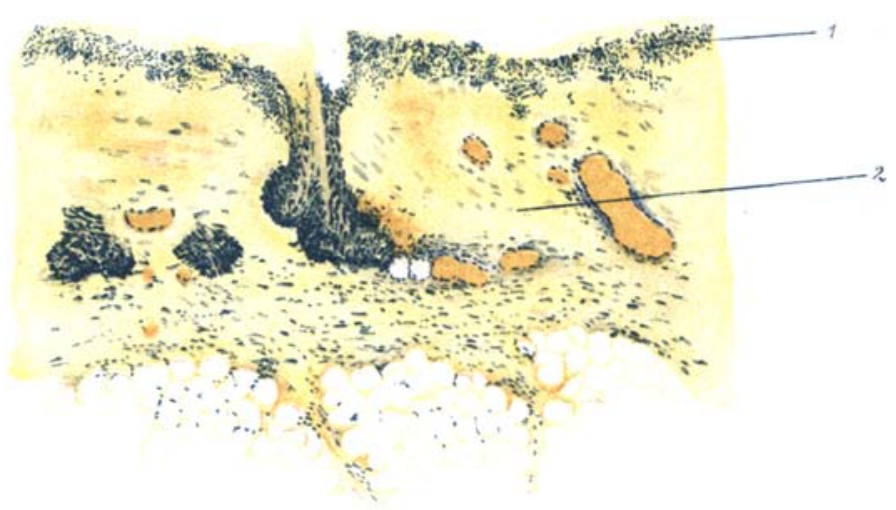

Hitschmann u. Kreibich : Ecthyma gangraenosum. 